\title{
Stretch reflexes of triceps surae in patients with upper motor neuron syndromes
}

\author{
A BERARDELLI, * AF SABRA, M HALLETT, W BERENBERG, SR SIMON \\ From the Rehabilitation Engineering Center of Harvard, MIT, Section of Neurology, Department of Medicine, \\ Brigham and Women's Hospital, Departments of Pediatrics and Orthopedic Surgery, Children's Hospital \\ Medical Center and Departments of Neurology, Pediatrics and Orthopedic Surgery, Harvard Medical School, \\ USA
}

SUMMARY Electromyographic responses of triceps surae to dorsiflexion stretch were studied in 47 patients with a variety of lesions producing an upper motor neuron syndrome. The short latency spinal reflexes, both when the patient was at rest and when he was exerting a voluntary plantarflexion, were frequently enhanced in magnitude and the rate of increase with acceleration was also enhanced. Long-latency reflexes were uncommon at rest. With background force longlatency reflexes were present unless the short latency reflex was very large. Long latency reflexes often were normal, but in some patients they were either excessively large or even of abnormal shape with prolonged continuous activity. The clinical assessment of the ankle jerk correlated with the magnitude of the short latency reflex. The clinical assessment of tone correlated with the magnitude of the short latency reflex, the magnitude of the long latency reflex and the duration of the long latency reflex. There appear to be multiple physiological mechanisms underlying the clinical phenomenon of spasticity.

The upper motor neuron syndromes, which arise from injury to the sensorimotor cortex, internal capsule, pyramidal tract and other descending motor pathways, are usually characterised by spasticity. Spasticity describes a type of increased tone, but the term is used to describe several different conditions in which there is an exaggerated response to stretch. There is an increased phasic response to stretch, at least one component of which is the monosynaptic reflex which has its clinical counterpart in enhanced tendon jerks and clonus. Tonic stretch reflexes also are enhanced and the clinical counterpart of this is spasticity. The discovery of long-latency reflexes to phasic stretch of muscle ${ }^{1-3}$ has raised questions about the possible relationship of these phenomena to increased tone.

There has been some previous work on the behaviour of long latency reflexes in upper extremity *Present address: Laboratorio di Neurofisiologia, V Clinica
Neurologica, Università di Roma, Italy.

Address for reprint requests: Mark Hallett, MD, Section of Neurology, Brigham and Women's Hospital, 75 Francis Street, Boston, Ma. 02115 USA.

Received 20 November 1981 and in revised form 22 June 1982. Accepted 15 July 1982 muscles with the clinical upper motor neuron syndrome. Some of the patients had lost long-latency reflexes ${ }^{45}$ which was used as evidence for the notion that long-latency stretch reflexes of upper extremity muscles utilise a trans-cortical pathway. Long-latency reflexes in the triceps surae have not been identified until recently, ${ }^{6}$ and their behaviour is somewhat different from that of long-latency reflexes in upper extremity muscles.

In normal subjects stretch of the triceps surae at rest produces a single short latency reflex. Stretch of the muscle when the subject is exerting a voluntary plantarflexion force produces a short-latency reflex followed by one and sometimes two later responses. ${ }^{6}$ We have explored the relationship between increased tendon jerks and spastic tone with short and longlatency stretch reflexes in this muscle group.

\section{Methods}

The general arrangements for studying the EMG responses of triceps surae and tibialis anterior have been extensively described in previous papers. ${ }^{67}$ The subject sat in a chair with the hip and knee flexed at $90^{\circ}$ and one foot strapped to a platform. A strain gauge was incorporated in the platform, so that the force exerted by the subject was measured and 
could be monitored by the subject on a meter. A torque motor displaced the platform and was controlled by a PDP $11 / 10$ computer. The platform reached the specified velocity in approximately $50 \mathrm{~ms}$ and dorsiflexed the ankle from -10 degrees to +5 degrees at random times at one of three velocities $(100,150,200$ degrees/s). Since peak velocity was attained more quickly with faster velocities, acceleration was a better single describer of the mechanical event than the specified velocity itself. Hence the results are reported in terms of acceleration. Occasionally slow velocities of stretching have been used. Each velocity was repeated ten times and the results were separately averaged. Surface EMG electrodes with preamplifiers at the site of recording were positioned on triceps surae and tibialis anterior. The EMG was rectified and filtered before collection. The EMG signal, angular position of the ankle and the force exerted on the platform were sampled by the computer at $2 \mathrm{~ms}$ intervals. The latency of EMG responses was measured from the electronic command to move the pedal, the duration by visual inspection and the magnitude of the EMG activity was measured by integration. The integrated EMG value was normalised to the EMG activity obtained at maximum voluntary effort (method 2 from ref 6 ). Hence activity equalling maximum voluntary effort was given a value of 100 . The subjects were studied using two different experimental conditions. In the first there was no background torque exerted by the patient on the platform. In the second the subject voluntarily exerted a plantarflexion torque on to the platform of $20 \%$ of his maximum effort while waiting for the perturbation.

The study was performed on 47 patients who gave informed consent to participate, 16 with multiple sclerosis, 11 with cerebral palsy, 7 with stroke, 6 with cervical spondylosis, 2 with cortical tumours, 2 with amyotrophic lateral sclerosis, 1 with primary lateral sclerosis, 1 with chronic myelopathy and 1 with olivo-ponto-cerebellar degeneration. Each patient was examined by the experimenters and the clinical signs were quantified. Strength was evaluated by the $0-5$ scale described in the Medical Research Council Memorandum (Aids to the Examination of the Peripheral Nervous System). Tone was classified as normal (0), mild (1), moderate (2) or marked (3). Reflexes were classified as absent (0), reduced (1), normal (2), increased (3) and markedly increased, often with clonus (4).

\section{Results}

\section{Short-latency stretch reflex}

In all the patients there was a prominent short-latency reflex when the triceps surae was stretched at rest (fig 1). The latencies measured from the electronic command to move the pedal varied from 40 to $85 \mathrm{~ms}$ and were shorter with more rapid stretch. The durations varied from 30 to $70 \mathrm{~ms}$ and were longer than those reported in normal subjects. ${ }^{6}$ The magnitude of the short-latency reflex for each patient was the value of the EMG at $3600 \mathrm{deg} / \mathrm{s}^{2}$ acceleration which was obtained from linear interpolation from the actual data points (fig 2). Without background

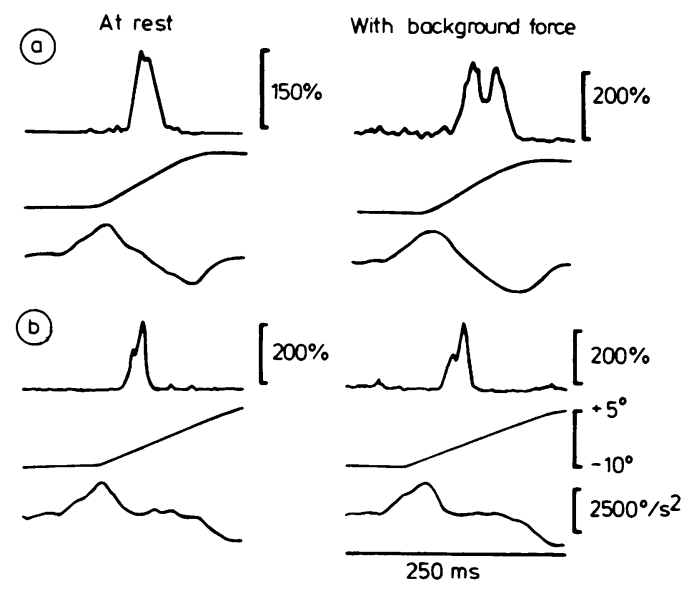

Fig 1 EMG responses of lateral gastrocnemius muscle in two patients, $A$ and $B$, with multiple sclerosis. The left side of each part shows the response at rest and the right side shows the response with background force. In each part of the figure the three traces from above downward are: rectified EMG, ankle angle and ankle angular acceleration, and each record is the average of 10 trials. The patient in A develops longlatency reflex with background force while the patient in $B$ does not.

force the short-latency reflex was enhanced in 37 of the 44 patients studied, and this enhancement was not due to an increase of the baseline EMG activity prior to the stretch, since this was similar to that of normal subjects. In general the magnitude of the shortlatency reflex increased with increased acceleration of the stretch. In order to quantify the rate of increase of magnitude with increased acceleration, the slope of the least-square straight line was taken as an approximation. The short-latency reflex increased with faster acceleration of stretches in all; the rate of increase was enhanced in 28 of 42 patients at rest. The patients with the highest rates of increase tended to be the same as those with the largest absolute magnitudes, and the coefficient of correlation between these two parameters for all of the patients was $0 \cdot 83$.

When the subjects exerted a background force on the pedal, the short-latency reflex remained prominent with slightly shorter latencies than at rest and again increased in magnitude with increased acceleration of stretch. The magnitude was enhanced in 30 of the 45 patients studied, and the rate of increase with acceleration was enhanced in 22 of 45 patients (fig 2). The short-latency reflex of 29 patients increased in magnitude when a background force was voluntarily exerted, but in 15 there was no increase. There was not much difference between patient groups, but those with strokes and tumours showed more normal short-latency reflexes. 

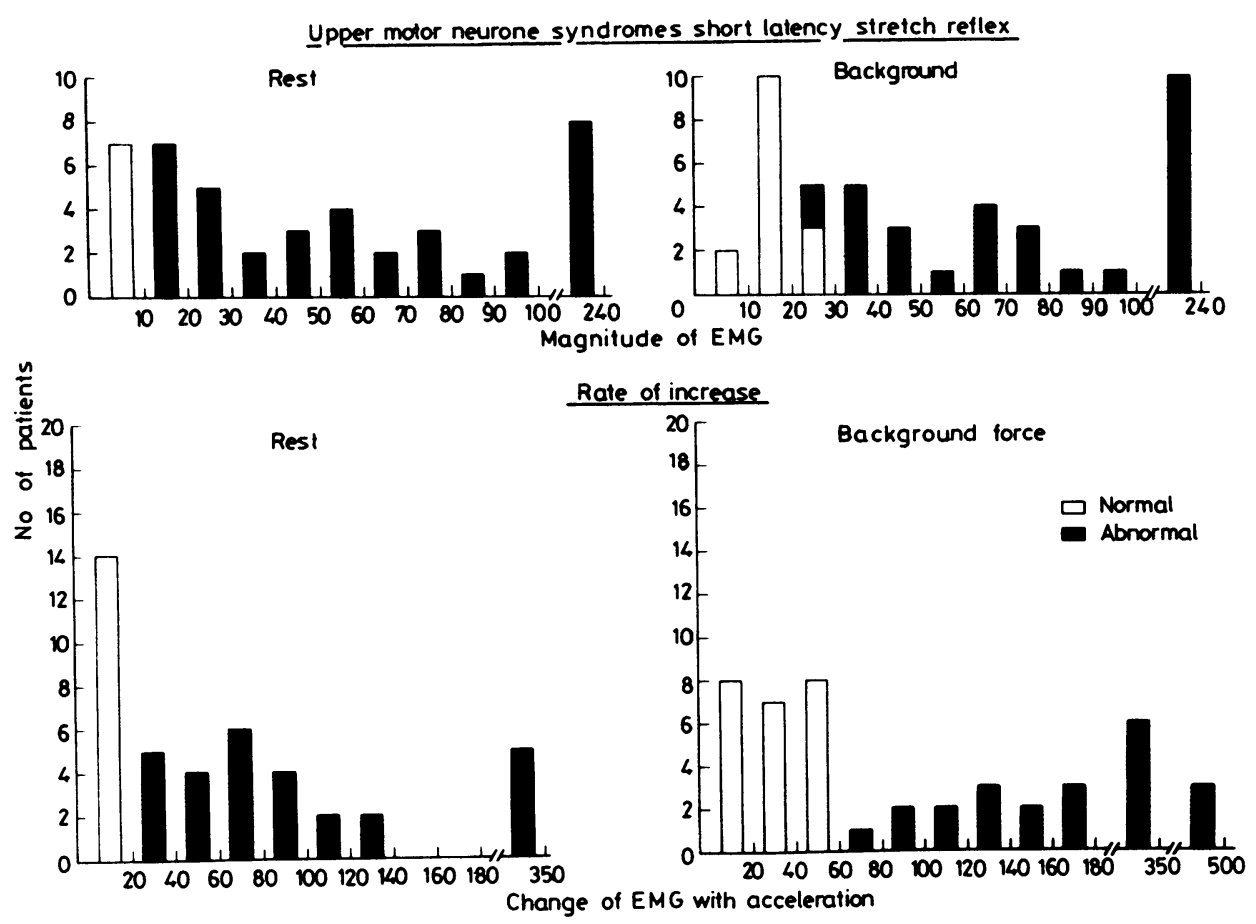

Fig 2 (Top) Number of patients with specified EMG magnitude for short-latency reflex at rest and with $20 \%$ background force. (Bottom) Number of patients with specified rate of increase of short-latency reflex magnitude with acceleration at rest and with background force. See text for details. Light bars are normal and dark bars are abnormal.

Long-latency stretch reflexes

Long-latency reflexes were recorded in only three of 47 patients at rest, one with a stroke, one with a brain tumour and one with primary lateral sclerosis. The patient with primary lateral sclerosis had the most marked tone of any patient studied and had large long-latency reflexes at rest. The appearance of the long-latency reflexes at rest could not be ascribed to inadequate relaxation since the background EMG was close to zero.

When the patients exerted a background force, long-latency stretch reflexes appeared (figs 1,3) and showed different behaviour which could be classified into three different groups (fig 4). The long-latency reflexes were absent for all accelerations in the first group which included 11 of the 47 patients. All these patients had enhancement of their short-latency reflex compared to normal (EMG value with background torque greater than 114). A second group of 25 patients showed a single long-latency discrete component or two discrete components with normal appearance, duration and amplitude. All these patients had an enhancement of the shortlatency reflex but less than that observed in the group with absent long-latency reflexes (with one exception all less 114). The long-latency reflexes tended to plateau or decrease (or disappear) with faster acceleration which is the normal behaviour. ${ }^{6}$ The third group of 15 patients was characterised by having large long-latency reflexes. There were seven patients
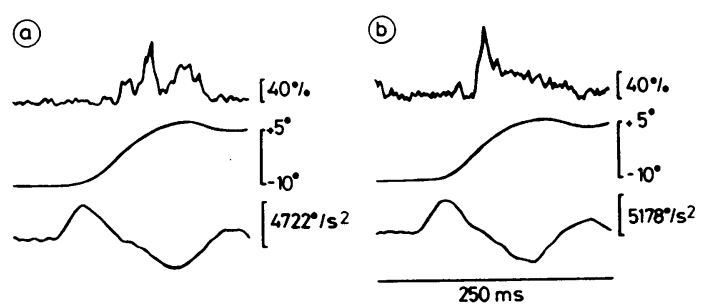

Fig 3 EMG responses of lateral gastrocnemius muscle on the normal side $(A)$ and on the abnormal side $(B)$ of a patient with cervical spondylosis exerting background force. The traces from above downward are rectified EMG, ankle angle and ankle angular acceleration, and each represents the average of 10 trials. The normal side shows two distinct long-latency reflexes while the abnormal side shows a long continuously delaying response. 


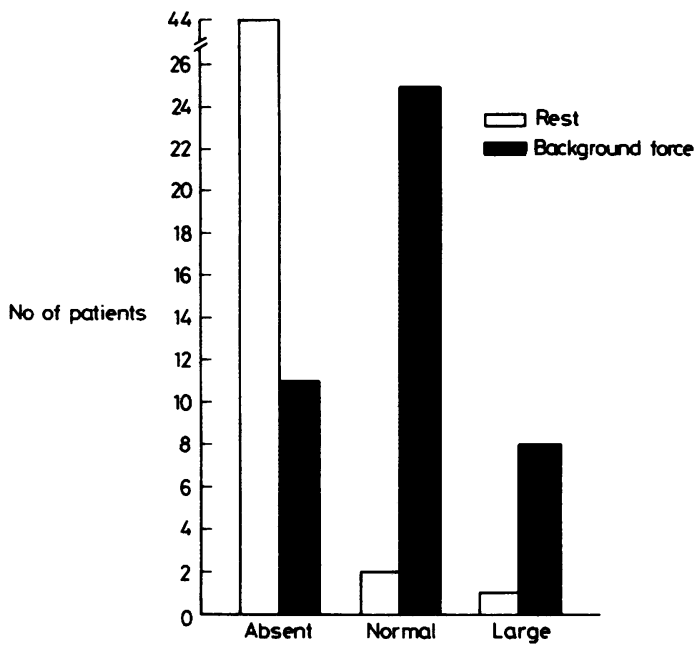

Fig 4 Number of patients with different types of longlatency reflexes at rest (light bars) and with $20 \%$ background force (dark bars).

with long-latency reflexes that had durations longer than just a single component that was not easily divisible into two different responses (fig 3 ). This long continuous component did not have a typical configuration for normal long-latency reflexes. The amplitude of these long-latency reflexes was within the normal range and showed little change with different velocities of stretch. Eight patients showed an increase in magnitude of a single discrete longlatency reflex (fig 1A). Some of the patients even demonstrated an apparent increased rate of enhancement of the long-latency reflex with faster accelerations. The short-latency reflex was normal or mildly increased for all patients. It can be noted that the three patients with long-latency reflexes at rest all fell into this group.

Considering the behaviour of the long-latency reflexes for the different groups of patients it can be noted that the absence of the long-latency reflexes was mainly observed in the patients with multiple sclerosis. Enhancement of the long-latency reflexes was present in some of the patients in each group.

\section{EMG responses with slow velocity of stretching}

Eleven patients have been studied at rest with slow stretching of triceps surae. No substantial continuous EMG responses have been recorded in ten of these patients with velocity of stretches varying from 15 to $30 \%$. A small phasic EMG response appeared at faster velocities which progressively increased in magnitude with increased velocity of stretching. Sometimes clonus was produced. In one patient (the one with primary lateral sclerosis) there was tonic EMG activity with velocity of stretching varying from $10^{\circ}$ to $30 \%$ (increasing the velocity of stretch beyond $30 \%$ a phasic response appeared and the tonic EMG activity disappeared). If a fast plantar flexion $\left(200^{\circ} / \mathrm{s}\right)$ followed the slow dorsiflexion the tonic EMG activity disappeared at a latency of 60-70 ms. This patient showed a marked hypertonia with increase of reflexes and had marked increase in long-latency reflexes including a long-latency reflex at rest.

In five patients the EMG responses with background force have been studied varying the velocity of stretches from $30^{\circ}$ to $100^{\circ} / \mathrm{s}$. In two patients with absent long-latency reflex at usual velocities we did not observe any long-latency reflexes decreasing the velocity of stretching even until the disappearance of the short-latency reflex. Three patients with only a long-latency reflex at usual velocities have been studied. In two of these only a single EMG component continued to be recorded, while in the third patient the long-latency reflex increased in duration with slower velocities so that two discrete long-latency reflexes could be easily recognised.

\section{Clinical correlation}

In attempting to correlate the clinical signs and physiological results we made formal correlations using chi square tests between the different parameters. For the purpose of these tests we divided tone in two levels (1) normal and mildly increased and (2) moderately and markedly increased. Tendon reflexes were divided into (1) reduced and normal and (2) increased. Short-latency reflex was divided into either (1) normal or (2) large, and the response was considered large if a response at any acceleration was greater than the normal range. Long-latency reflexes were looked at for different purposes in two different ways. A division was made on the basis of magnitude into either (1) absent, (2) normal and (3) large and the response was considered large if a response at any acceleration was greater than normal range. A second method for analysis of the reflexes was on the basis of their durations. The first important result was that tone and tendon jerks were correlated with each other $(\mathrm{p}<0.025)$. This makes some difficulty in trying to separate the physiological parameters that underlie each of these two signs.

The magnitude of the short-latency reflex was correlated with the size of the tendon jerk, somewhat better with the short-latency reflex at rest $(p<0$ - 005) than with background force $(p<005)$. The correlation was less good with tone $(p<0.025$ for short-latency reflex at rest; $p<0.25$ for background force). The correlation of short-latency reflex and tendon jerk was certainly understandable in terms of 
the known physiology of the short-latency reflex which suggests that it is similar to the tendon jerk.

It becomes difficult to analyse the long-latency reflexes because it appears that their amplitude may depend in part on the amplitude of the short-latency reflex which precedes them. Indeed there was one group of patients who had no long-latency reflexes at all; it has been pointed out that the short-latency reflex was large in all these patients. If it really is the case that a large short-latency reflex inhibits any possible long-latency reflex, then the group without long-latency reflexes cannot be analysed because the long-latency reflexes are suppressed. Therefore we did not consider this group in the clinical correlations of the long-latency reflexes. Additionally, even if the long-latency reflexes were present they may have been reduced in amplitude because of the shortlatency reflex, so correlations of the long-latency reflexes were possibly underestimated. We attempted to correlate the presence or absence of the longlatency reflexes at rest with the two levels of tone, but no correlation was demonstrated $(p<0.750)$, perhaps because of the rare appearance of longlatency reflexes at rest. On the other hand, the magnitude of long-latency reflexes with background force did correlate with tone $(p<0.025)$. The average duration of the long-latency reflexes with normal or mild tone was $32 \mathrm{~ms}$, and with moderate or marked tone was $45 \mathrm{~ms}$, this difference was statistically significant $(p<0.05)$. There may have been a difference in the quality of the increased tone in the two groups (increased magnitude and long duration with atypical configuration), but this was not apparent by clinical evaluation or by consideration of the etiologies.

\section{Discussion}

A short-latency reflex has been recorded in all the patients, with and without background force, and its magnitude and rate often was enhanced. The shortlatency reflex of the triceps surae appears to be a monosynaptic stretch reflex ${ }^{68}$ and the increase of short-latency reflex in these patients parallels the well-known increase of the anke-jerk. This enhancement of phasic stretch reflexes is a typical feature of the upper motor neuron syndrome and seems not due to enhanced spindle afferent activity. ${ }^{9-11}$ The alpha motor neurons themselves must have an enhanced response to a normal afferent input as suggested by Landau and Clare. ${ }^{12}$

This velocity-dependent behaviour in the upper motor neuron syndrome has been observed also by Herman ${ }^{13}$ and by Burke et al $^{1415}$ in hamstrings and quadriceps muscles and in biceps and triceps brachii by Ashby and Burke. ${ }^{1617}$ Enhancement of rate of growth of short-latency reflex with acceleration is somewhat more objective than is enhancement of absolute magnitude, since determination of absolute magnitude depends on assessment of maximum voluntary force which might be influenced by patient cooperation. The fact that these two parameters are correlated suggests that absolute magnitude is a reasonable measure. The short-latency reflex did not always increase in magnitude when a background force was voluntarily exerted. This lack of increase was often observed when the short-latency reflex was already large in magnitude at rest. This phenomenon is not normal, ${ }^{6}$ but has been observed before by Neilson ${ }^{18}$ in patients with the upper motor neuron syndrome.

The behaviour of the long latency reflexes has been classified into three different groups. In the first, the long-latency reflexes were absent and a large shortlatency reflex was present. One possible explanation for this phenomenon is that if the motor neuron pool produced a large monosynaptic response then it is not able to produce responses to subsequent stretch stimuli. In normal subjects ${ }^{6}$ the first long-latency reflex plateaued and the second long-latency reflex tended to decrease as the short-latency reflex increased with increased acceleration of stretch. In addition, a small $\mathrm{H}$ reflex prior to a stretch did not abolish the response to stretch whereas a large $\mathrm{H}$ reflex did abolish the response to stretch. Possible mechanisms for this type of inhibition include hyperpolarisation of motor neurons, Renshaw cell activity and sensory feedback. ${ }^{19}$ It is conceivable therefore that the absence of the long-latency reflex reflects a period of inhibition following a large monosynaptic response. In accord with this hypothesis is the behaviour of the long-latency reflexes in some of the patients in other groups who exhibited complete disappearance of this component at faster accelerations while the short-latency reflex was growing. We attempted to gather more evidence for this notion by studying some of the patients at velocities of stretch slower than the standard ones, hoping to reduce the magnitude of the short-latency reflex sufficiently so that we might expect to see a long-latency reflex if none was present before. Experiments performed in two patients with no longlatency reflexes with the usual stretches showed that the long-latency reflex did not appear with slower stretches, but at least in one patient the magnitude even at its threshold was enhanced with respect to short-latency reflexes of normal subjects with usual accelerations. Additional experiments with slower stretches were carried out on four patients with one long-latency component attempting to produce a second long-latency component; one did show the development of a second long-latency component in addition to the first long-latency component. 
Absence of long-latency reflexes therefore seems explicable to a certain extent on the basis of enhanced short-latency reflex. Another important possibility was that the lesion that has caused the upper motor neuron syndrome had disrupted the pathway for the long-latency reflexes. This possibility has been raised before in relation to long-loop pathways ${ }^{2-50-22}$ but the apparent high proportion of presumed spinal lesions as compared to presumed cortical lesions in our patients with absent long-latency reflexes suggests the importance of pathways limited to the spinal cord.

The second group of patients had a single or two discrete components with normal appearance, duration and amplitude and normal velocity behaviour. The short-latency component was increased but to a lesser extent than the first group.

The third group of patients was characterised by having large long-latency stretch reflexes while the short-latency reflex was normal or mildly increased. Patients in this last group demonstrated either exaggerated normal long-latency behaviour or superimposition of a new mechanism on top of the normal mechanism. We do not have much data to understand these mechanisms. It appears that the subgroup with the long continuous long-latency reflex has the superimposition of some kind of tonic response. The sub-group with the enhanced first component of the long-latency reflex appears to show an exaggerated normal response or the superimposition of a late phasic response. If this is in fact an exaggerated normal response and if the mechanism of the response is largely a second monosynaptic reflex (as we have suggested previously), ${ }^{6}$ then it would appear that the normal inhibitory mechanism following a monosynaptic reflex exhibited by the first group is diminished in these patients.

Experiments with the slow ramp stretches were undertaken to learn more about abnormal physiological mechanisms of the responses to stretch in these patients. Most of these studies produced only phasic responses similar to the short-latency reflex revealing a low acceleration threshold for this response in these patients. One patient with marked increase in tone whose long-latency reflexes were characterised by a large long-latency first component of increased magnitude did show tonic activity with the ramp stretch. When stretching was suddenly stopped the tonic activity disappeared at a latency similar to the latency of the short-latency reflex after stretch. This suggests that the tonic activity is strongly supported by activity in the monosynaptic pathways. This also might be taken as evidence for the idea suggested above that patients with the abnormally large long-latency first component may have enhanced monosynaptic responses.
Marsden $e t a l,{ }^{4}$ found in their patients with upper motor neuron syndrome that the long-latency responses of long flexor of the thumb were diminished or abolished in 13 of 14 patients. This contrasts with our results where only 11 of 47 patients had absent long-latency reflexes and only one of nine patients with a cerebral stroke or tumour (similar to cases of Marsden et $\mathrm{al}^{4}$ ) had absent longlatency reflexes. Our results are apparently similar to the preliminary data of Lee and Tatton. ${ }^{5}$ These different results could be explained by several possible mechanisms. The patients of Marsden et al ${ }^{4}$ were mostly in an acute stage of their illness while many of our patients were in a chronic phase. In one of their patients the long-latency reflexes became larger with the passage of time. It is also possible that the spectrum of the sites of the lesions in the central nervous system differ in two groups and that with these different lesions the pathophysiology might differ. Another possibility is a difference in basic mechanism between long-latency reflexes in the long flexor of the thumb and in the triceps surae. In a previous work $^{6}$ we have investigated the physiological mechanisms of the long-latency reflexes of the triceps surae and pointed out differences to the long-latency reflexes of the long flexor of the thumb. We have suggested that a possible reason for this difference is that while the long-latency reflexes of the thumb and other upper extremity muscles may be largely dependent on long-loop supraspinal pathways, ${ }^{12520-22}$ the long-latency reflexes of triceps surae may depend mostly on spinal pathways.

The behaviour of the stretch reflexes of triceps surae with slow and phasic stretches in subjects with upper motor neuron syndromes is different from that observed by us in a group of patients with rigidity due to Parkinson's disease. ${ }^{7}$ In patients with rigidity the baseline activity could be high and the short-latency reflex was in general normal or only mildly increased. The long-latency reflexes often appeared when the patients were trying to relax and were usually enhanced in magnitude when a background force was voluntarily exerted. In addition the long-latency reflexes were prolonged and often merged with subsequent EMG activity which was in the range of voluntary reaction time, and slow ramp stretches often produced tonic activity.

\section{Clinical correlation}

Our results show a clear relationship between the tendon jerk and the short-latency reflex magnitude and rate. The short-latency reflex is more objective than the routine clinical measure and would be a useful parameter in any quantitative assessment of the phasic stretch response of spastic patients. Understanding the tonic stretch response of spasticity 
is more difficult. Despite the fact that our standard stretch is maintained for approximately $3 \mathrm{~s}$ most of these patients really just show phasic responses, only a short-latency reflex or a short-latency reflex and brief long-latency reflexes similar to normal subjects. After these responses the muscle is silent to the end of our recording. Attempts to produce continuous responses with ramp stretches usually produce only phasic responses or a series of phasic responses (clonus); in only one patient of 11 was there a true continuous response. Perhaps some of the clinical impression of tonic resistance to stretch comes in fact from increased phasic responses which may appear at lower thresholds of acceleration than would be expected normally.

It would seem from this data that long-latency reflex behaviour certainly also makes a contribution to spastic tone. Some patients show long-latency reflexes which are increased in magnitude and others show long-latency reflexes increased in duration and both of these phenomena correlate with the clinical impression of tone. All this illustrates the fact, which is really apparent clinically, that there is more than one type of spastic tone and they do not have a homogeneous aetiology. This type of study is a useful window into the clinical phenomena and provides a qualitative and quantitative assessment of the pathophysiology. The results confirm and extend the view that there are multiple mechanisms for the longlatency reflexes some of which may only appear in pathological states.

The work was supported by a grant to the Rehabilitation Engineering Center by the NIHR (23P-55854/1) and a grant from the Whittaker Health Science Fund of MIT. R Ackerman provided technical support. Alfredo Berardelli was supported by a fellowship from Consiglio-Nazionale Delle Richerche (CNR), NATO, Italy and from the Brigham and Women's Hospital Amyotrophic Lateral Sclerosis Research Fund.

\section{References}

${ }^{1}$ Hammond PH. An experimental study of servo action in human muscular contraction. Proc III Int Conf on Medical Electronics. London: Institution of Electrical Engineers 1960;190-9.

${ }^{2}$ Marsden CD, Merton PA, Morton HB. Servo action in human voluntary movement. Nature 1972;238:140-3.

${ }^{3}$ Lee RG, Tatton WG. Motor responses to sudden limb displacement in primates with specific CNS lesions and in human patients with motor system disorders. Can J Neurol Sci 1975;2:285-93.

${ }^{4}$ Marsden CD, Merton PA, Morton HB, Adam JER. The effect of lesions of the sensorimotor cortex and the capsular pathways on servo responses from the human long thumb flexor. Brain 1977;100:503-26.

${ }^{5}$ Lee RG, Tatton WG. Long loop reflexes in man: Clinical applications. In: Desmedt JE, ed. Progress in Clinical Neurophysiology. Basel: Karger, 1978;4:320-33.

${ }^{6}$ Berardelli A, Hallett M, Kaufman C, Fine E, Berenberg W, Simon S. Stretch reflexes of triceps surae in man. $J$ Neurol Neurosurg Psychiatry 1982;45:513-25.

${ }^{7}$ Berardelli A, Sabra AF, Hallett M. Physiological mechanisms of rigidity. J Neurol Neurosurg Psychiatry 1982. (In press.)

${ }^{8}$ Gottlieb GL, Agarwal GC. Response to sudden torques about ankle in man: Myotatic Reflex. $J$ Neurophysiol 1979;42:91-106.

${ }^{9}$ Hagbarth KE. The effect of muscle vibration in normal man and in patients with motor disorders. In: Desmedt JE, ed. New Developments in Electromyography and Clinical Neurophysiology. Basel: Karger, 1973;3:42843.

${ }^{10}$ Hagbarth KE, Wallin G, Lofstedt L, Aquilonius SM. Muscle spindle activity in alternating tremor of Parkinsonism and in clonus. J Neurol Neurosurg Psychiatry 1975;38:636-41.

$"$ Gilman S, Lieberman JS, Marco LA. Spinal mechanisms underlying the effects of unilateral ablation of areas 4 and 6 in monkeys. Brain 1974;97:49-64.

${ }^{12}$ Landau WM, Clare MH. Fusimotor function. Part VI. $H$-reflex, tendon jerk and reinforcement in hemiplegia. Arch Neurol 1964;10:128-34.

${ }^{13}$ Herman R. The myotatic reflex. Clinico-physiological aspects of spasticity and contracture. Brain 1970;93:273-312.

${ }^{14}$ Burke D, Gillies JB, Lance JW. Hamstrings stretch reflex in human spasticity. J Neurol Neurosurg Psychiatry 1970;34:231-5.

${ }^{15}$ Burke D, Gillies JB, Lance JW. The quadriceps stretch reflex in human spasticity. $J$ Neurol Neurosurg Psychiatry 1970;33:216-23.

${ }^{16}$ Burke D, Andrews C, Gillies JD. The reflex response to sinusoidal stretching in spastic man. Brain 1971;94:445-70.

${ }^{17}$ Ashby P, Burke D. Stretch reflexes in the upper limb of spastic man. J Neurol Neurosurg Psychiatry 1971;34:765-71.

${ }^{18}$ Neilson PD. Voluntary and reflex control of the biceps brachia muscle in spastic-athetotic patients. $J$ Neurol Neurosurg Psychiatry 1972;35:589-98.

19 Andressen S, Rosenfalck A. Regulation of the firing pattern of single motor units. J Neurol Neurosurg Psychiatry 1980;43:897-906.

${ }^{20}$ Phillips CG, Powell TPS, Wiesendanger M. Projection from low-threshold muscle afferents of hand and forearm to area 3a of baboon's cortex.J Physiol (Lond) 1972;217:419-46.

${ }^{21}$ Marsden CD, Merton PA, Morton HB. Servo action in the human thumb. J Physiol (Lond) 1976;257:1-44.

${ }^{22}$ Marsden CD, Merton PA, Morton HB, Adam JER, Hallett M. Automatic and voluntary responses to muscle stretch in man. In: Desmedt JE, ed. Progress in Clinical Neurophysiology. Basel: Karger, 1978;4:16777. 